\title{
RNA interference approaches for plant disease control
}

\author{
Yen-Wen Kuo ${ }^{\ddagger} 1$ (iD) \& Bryce W Falk*,‡,1
}

${ }^{1}$ Department of Plant Pathology, University of California, Davis, CA 95616, USA; *Author for correspondence: bwfalk@ucdavis.edu; ${ }^{\ddagger}$ Authors contributed equally

BioTechniques 69: 469-477 (December 2020) 10.2144/btn-2020-0098

First draft submitted: 7 July 2020; Accepted for publication: 2 September 2020; Published online: 19 October 2020

\begin{abstract}
Plant diseases caused by a variety of pathogens can have severe effects on crop plants and even plants in natural ecosystems. Despite many effective conventional approaches to control plant diseases, new, efficacious, environmentally sound and cost-effective approaches are needed, particularly with our increasing human population and the effects on crop production and plant health caused by climate change. RNA interference (RNAi) is a gene regulation and antiviral response mechanism in eukaryotes; transgenic and non transgenic plant-based RNAi approaches have shown great effectiveness and potential to target specific plant pathogens and help control plant diseases, especially when no alternatives are available. Here we discuss ways in which RNAi has been used against different plant pathogens, and some new potential applications for plant disease control.
\end{abstract}

\section{KEYWORDS:}

host-induced gene silencing • miRNA • pathogen $\bullet$ RNA-induced silencing complex • RNAi $\bullet$ siRNA

The Food and Agriculture Organization of the United Nations has declared 2020 as the International Year of Plant Health (http://www.fao. org/plant-health-2020/about/en/). This is important and very timely. The organization estimates that plant pests and pathogens cause annual losses of up to $40 \%$ for global food crops, leaving millions of people without enough food and causing drastic economic effects, particularly in rural areas where subsistence farming is a way of life. With our global population continuing to increase, these effects will be greater. Furthermore, the probability is good that at least some plant diseases will become more severe with ongoing climate change [1], reinforcing the need now for more research to understand plant-pathogen interactions and to develop efficient, economically affordable, environmentally sound approaches to controlling plant diseases.

Essentially all plants that humans grow for food and/or fiber can be affected by specific plant diseases. Various plant diseases can be caused by a range of different pathogens, including viruses, prokaryotes (bacteria and mollicutes), fungi, oomycetes, nematodes and even parasitic plants [2]. Several different approaches are used in attempts to help manage plant diseases and these vary by the plant, the pathogen, the environment and geographic location where the plants are grown. Conceptual approaches for controlling plant diseases include: avoidance - growing crop plants in areas where the pathogen does not occur; exclusion - preventing pathogen introduction into new areas; eradication - elimination of the pathogen if it is present in an area used for crop production; and protection - using strategies including pathogen-targeting chemicals and host plant genetic resistance to interfere with the pathogens' ability to infect plants and cause disease [2]. As with diseases of animals and humans (think of Covid-19), prevention or protection approaches are generally much more cost-effective and efficient in avoiding the devastating effects of plant diseases.

Protection by host plant resistance is generally considered to be the most desirable, environmentally sound strategy for controlling plant diseases [3,4], but too often we lack the ability to use resistance for plant disease control. This can be due to a number of reasons; for example, not having adequate sources of genetic resistance available for use in plant breeding programs, or the emergence of new pathogens for which resistance is not known. Even if good sources of genetic resistance are available, traditional plant breeding takes years, and plants having resistance to known pathogens might not be genetically compatible with the plants needing protection. Even with effective genetic resistance, we can expect that evolution may allow pathogens to be selected to take advantage of the new niche and overcome the resistance [5]. Because humans generally grow genetically uniform crops in large populations, if a virulent pathogen is present and environmental conditions are conducive for disease development the pathogen can spread rapidly and disease can be severe. Thus controlling plant diseases is an ongoing battle and new, effective, environmentally sound approaches are needed [4].

Research on plant-pathogen interactions has allowed a greater understanding of the molecular events that determine disease and/or resistance and has yielded opportunities to develop novel approaches to managing plant diseases. RNA interference (RNAi) approaches have already shown some successes, and ongoing efforts offer new opportunities to help control plant diseases.

\section{RNAi}

RNAi is a natural gene regulation mechanism in eukaryotes. It involves sequence-specific recognition of mRNAs and then repressing their expression either by ribonuclease-directed degradation or by preventing their translation. Two types of small single-stranded RNAs 


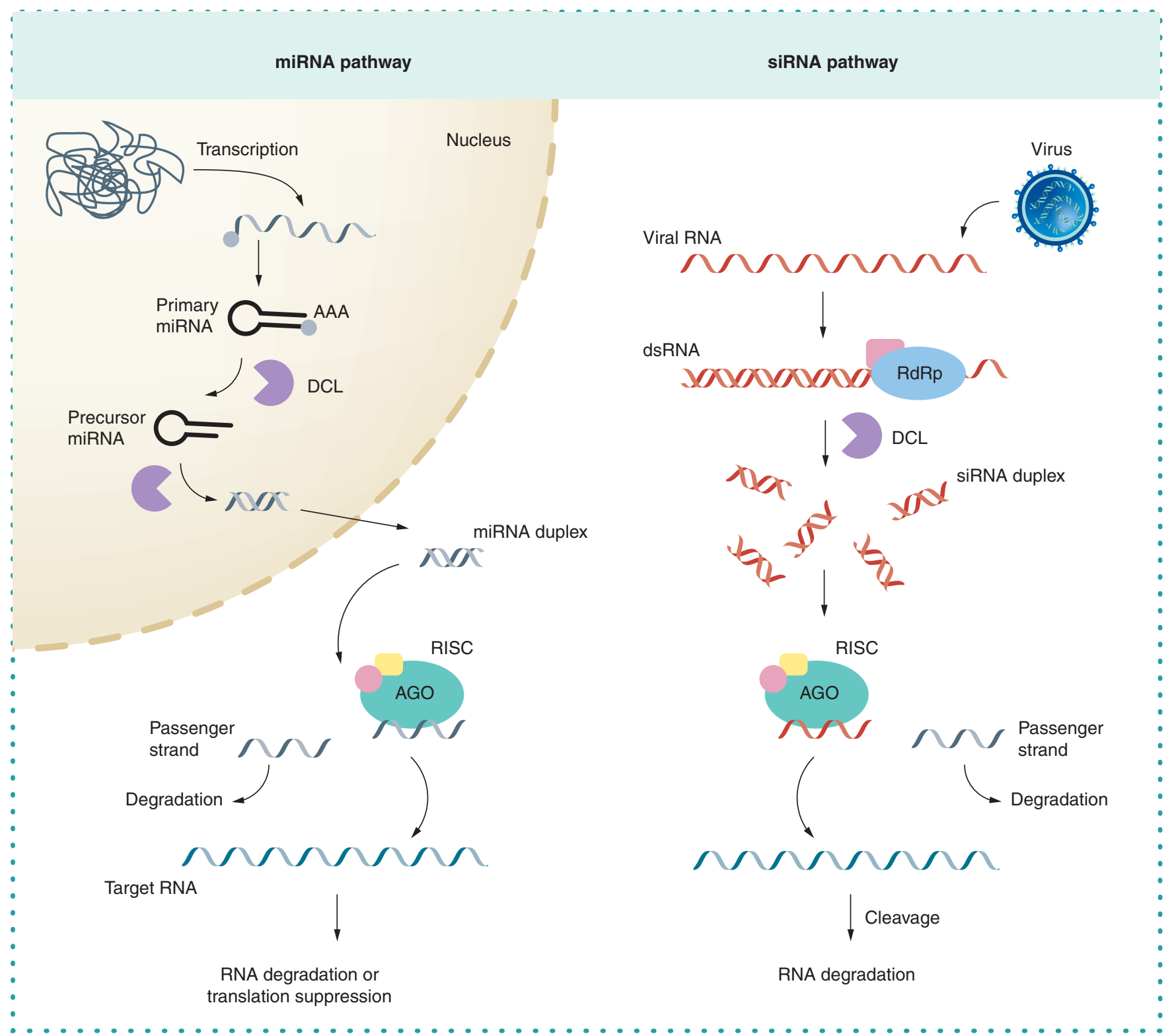

Figure 1. Model depicting the RNA interference miRNA and siRNA pathways within a plant cell. The miRNA pathway at left begins in the nucleus with transcription giving the hairpin folded primary miRNA (pri-miRNA). DCL in the nucleus first cleaves the pri-miRNA to give the precursor miRNA, which DCL cleaves to give the approximately 22-nucleotide miRNA duplex. The duplex is exported into the cytoplasm and becomes loaded into the RISC. One strand (the passenger strand) of the miRNA duplex is discarded and the other strand (the guide strand) is retained in RISC. When the RISC-bound guide RNA binds (by Watson-Crick base-pairing) to a target RNA, cleavage or translational arrest of the target results. The siRNA pathway begins in the cytoplasm when a target RNA (usually a structured aberrant RNA, or those of an infecting plant virus, as here) is recognized by DCL, resulting in cleavage of the target RNA into short (21-24 nucleotide) duplex RNAs. As for duplex miRNAs, one strand (passenger) is released and degraded and one (guide RNA) is retained by RISC. When the RISC-bound guide RNA binds to a target RNA, cleavage results. RNA-dependent RNA polymerase activity can amplify the RNAi response within the cell.

AAA: 3' polyadenylate tail; AGO: Argonaute protein; DCL: Dicer-like protein; Pri-miRNA: Primary microRNA; RdRP: RNA-dependent RNA polymerase; RISC: RNA-induced silencing complex; RNAi: RNA interference.

( 21-24 nucleotides) are key players in the two main RNAi pathways in plants; these are the siRNAs and miRNAs (Figure 1). miRNAs are encoded by the plant genome and mostly function as regulators of gene expression; siRNAs may also be derived from the genome, but plants have evolved to have an RNA surveillance system that recognizes and degrades specific RNAs, resulting in the generation of siRNAs. In plants this RNA surveillance system is also a robust antiviral defense mechanism.

The siRNA pathway in plants begins with recognition of RNAs for degradation (Figure 1). These RNAs are often dsRNAs or highly structured RNAs; they are recognized in the cell cytoplasm and targeted and cleaved by ribonuclease activity of Dicer-like proteins (DCLs). 
Different plants can contain different DCLs [6]; for example, Arabidopsis thaliana has four DCLs (DCL 1, 2, 3 and 4) [7], which cleave dsRNAs or the structured RNAs into small RNA duplexes (typically $21,22,23$ or 24 nucleotides). The beauty of the RNAi response is that the DCLgenerated small RNA duplexes are now recruited to the Argonaute protein (AGO), which is part of the RNA-induced silencing complex (RISC). One strand of the small RNA duplex is released (the passenger strand) and mostly degraded; the other is retained by AGO, and the retained strand now serves as a guide RNA. When the RISC, carrying the guide RNA, encounters an RNA that is homologous to the guide RNA (by Watson-Crick base pairing), AGO directs ribonuclease cleavage which results in degradation of the bound RNA. Furthermore, in plants, endogenous and virus-encoded RNA-dependent RNA polymerases can amplify the RNAi response $[6,8,9]$. Thus the siRNAi activity is highly specific and robust.

The majority of plant miRNAs originate from specific noncoding RNAs, also called primary microRNAs (pri-miRNAs), which fold into specific structures (Figure 1). Many of the MIRNA (MIR) genes are categorized into families based on their location in the genome and classified as intergenic or intronic. The intergenic miRNAs, located between coding genes, are transcribed independently by DNAdependent RNA polymerase II (Pol II), while the intronic miRNAs are processed from introns of RNA transcripts [10]. Pri-miRNAs are targeted by DCL in the nucleus. DCL processes the pri-miRNAs into precursor miRNAs and then processes the precursor miRNAs into the miRNA duplex ( $\sim 22 \mathrm{nt})$ in the nucleus. The miRNA duplex has 2-nt $3^{\prime}$ overhangs for both strands of the duplex and comprises the guide strand and the passenger strand. The duplex is exported into the cytoplasm and, similar to siRNAs, the mature miRNA (the guide strand) loads into RISC. The RISC-bound mature miRNA can guide the complex to target specific RNA sequences, resulting in translation suppression or RNA degradation.

Thus the siRNA and miRNA pathways are natural components of plants. They serve important roles in regulating gene expression but can also have important roles in host defense. There are a number of excellent reviews that clearly describe the details of these pathways [11-13].

\section{Transgenic plant-based RNAi strategies were first used to help control plant diseases caused by viruses}

The majority of plant-infecting viruses have single-stranded RNA as their genetic material and they replicate their genomes in the plant cell cytoplasm through dsRNA intermediates. Thus a conceptual approach which might be used to confer resistance against plant viruses is to pre-program plants to have an active RNAi defense against a particular viral RNA before the plant is challenged by that virus. Then, when the plant is challenged by the virus, the already active RNAi would recognize and degrade the incoming viral RNA before the virus can replicate and become established. Fortunately, plants can be genetically engineered to contain and express new genes, so engineering plants for specific antiviral RNAi-based resistance can be done.

Many plants can be genetically engineered by taking advantage of the common soil-inhabiting bacterium Agrobacterium tumefaciens, which naturally transfers genes into plant cells, causing the disease crown gall [2]. Agrobacterium tumefaciens contains a large plasmid, the Ti plasmid. When A. tumefaciens encounters a wounded plant cell, it transfers and inserts a portion of the Ti plasmid, the T-DNA, into the plant cell chromosome; the transformed plant cell then expresses the genes on the T-DNA [14]. The wild-type T-DNA contains genes encoding for plant hormones; expression of these genes in the transformed cells results in galls developing at the site of infection. But the Ti plasmid can be genetically engineered to remove the genes leading to gall formation (disarmed Ti plasmid) and modified instead to contain and express desirable genes; for antiviral resistance, this could mean engineering it to express RNA designed to induce RNAi activity toward the viral RNA genome. Agrobacterium tumefaciens can be used to transfer these genes into individual plant cells, and the individual transformed plant cells can be cultured in vitro to regenerate whole plants [15]. As each cell of the plant came from the original transformed cell, all cells will now contain the transgene in their chromosomal DNA, and it can be stable for generations.

Before RNAi was even recognized, Lindbo and Dougherty at Oregon State University genetically engineered plants to be resistant to tobacco etch virus (TEV) [16-18]. They engineered tobacco (Nicotiana tabacum) plants to transcribe the TEV coat protein mRNA, which would be translated in the cell to yield TEV coat protein, because the early approaches for virus resistance considered that the resistance would be protein-based. However, they included a very important negative control in their experiments: tobacco plants engineered to transcribe an untranslatable version of the TEV coat protein mRNA. The latter had essentially the same nucleotide sequence as that of the authentic TEV coat protein mRNA, but the untranslatable version contained a few nucleotide changes that introduced stop codons, preventing its translation and subsequent production of the TEV coat protein in those plants. The plants should still then produce the untranslatable TEV RNA, but it would not be translated to give TEV coat protein. When the TEV coat protein-expressing plants were inoculated with TEV, some resistance was noted. However, when challenged with TEV, some of the plants that had been engineered to produce the untranslatable RNA showed extreme resistance, or immunity. Careful experiments showed that nuclei isolated from the immune plants transcribed the transgene to give the untranslatable TEV coat protein mRNA, but the transcript did not seem to be present in the cytoplasm. The researchers concluded that the untranslatable RNA was degraded in the cytoplasm, post-transcriptionally; the interpretation was that the RNA sequence was recognized by something in the plant cell cytoplasm and specifically targeted for degradation. Because the TEV genomic RNA contained the same nucleotide sequence, when TEV RNA entered the cell after inoculation, it was now also recognized by the pre-programmed transgenic plant RNAi activity and was degraded, thereby conferring immunity to TEV (Figure 2A). This is RNAi-based antivirus immunity. 


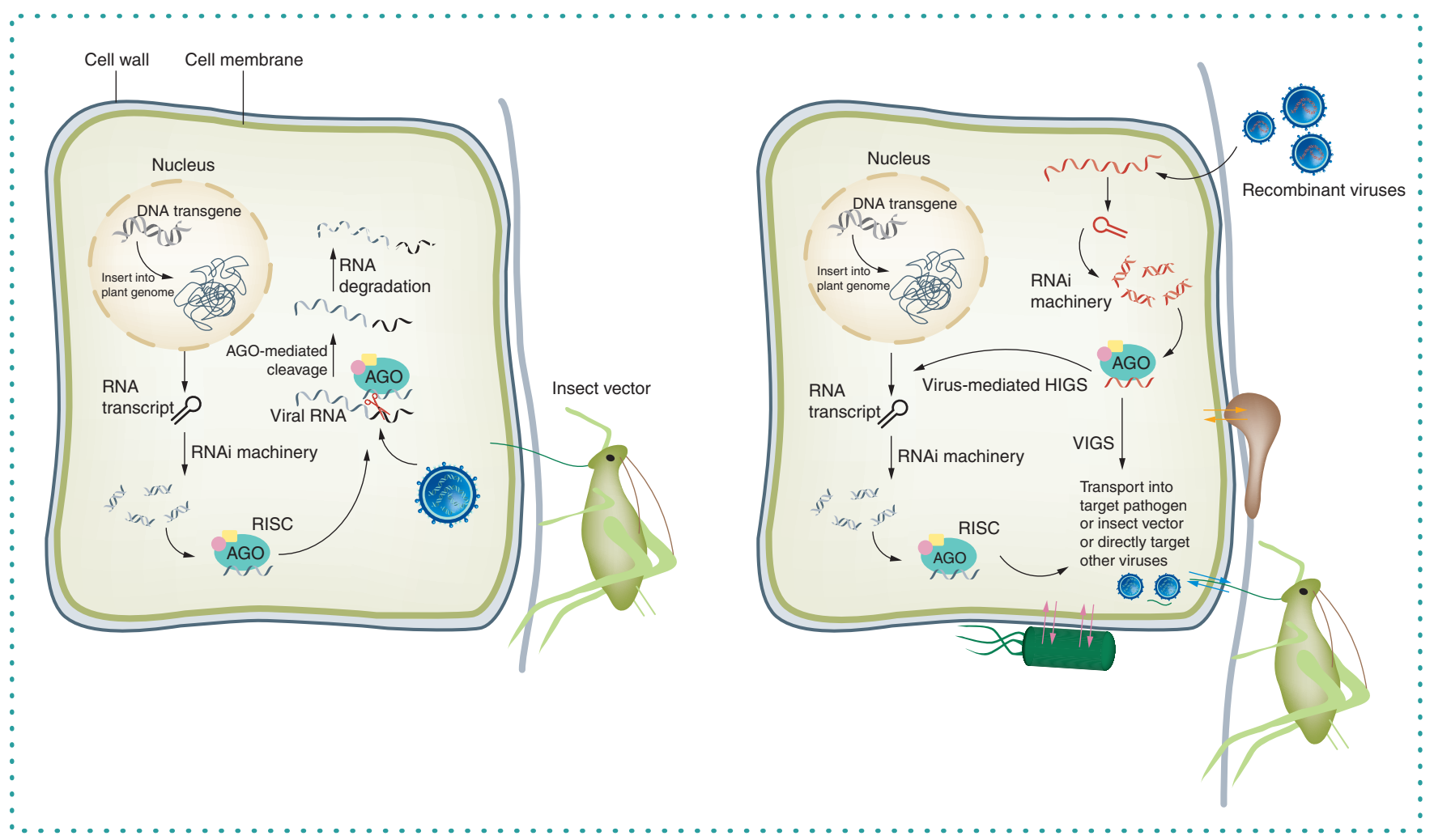

Figure 2. Models showing RNA interference activities toward plant pathogens and insect vectors of plant pathogens. (A) A model for transgenic RNAi-based antiviral resistance. The transgene is transcribed and the resulting transcript (typically a hairpin, partly double-stranded RNA) is exported to the cytoplasm. This RNA is recognized by the cytoplasmic RNAi machinery (see Figure 1 ) and degraded, and one strand (the guide strand) is incorporated into RISC. When the guide RNA in RISC recognizes and pairs with a homologous virus RNA, the virus RNA is degraded, resulting in resistance or immunity. (B) Transgenic and non transgenic host-induced gene silencing RNAi effects on plant pathogens and insect vectors of plant pathogens. RNAi is induced against a specific pathogen or insect vector mRNA in plants either transgenically, or by using a recombinant plant virus. This results in generation of siRNAs (or in some case, miRNAs) homologous to the target mRNA. These can be transported via different mechanisms into the corresponding pathogen (the brown structure on the right represents a germinating fungal spore; the small structure at the bottom is a bacterium) or insect vector (lower right), where they can induce RNAi effects against the specific mRNA. The arrows indicate that small RNA trafficking can go both ways: small RNAs can move from the plant into the pathogen/vector, but pathogens/vectors also transport small RNAs (and proteins) into plants to block plant defenses.

AGO: Argonaute protein; DCL: Dicer-like protein; HIGS: Host-induced gene silencing; RISC: RNA-induced silencing complex; RNAi: RNA interference; VIGS: Virus-induced gene silencing.

RNAi has proven to be an effective approach to controlling plant virus diseases for many different viruses in many plant species [12,19]. Instead of using untranslatable RNAs, plants are now mostly engineered to generate dsRNAs or structured RNAs, such as hairpin RNAs, as these are powerful inducers of RNAi [20-22]. However, despite the ability to engineer antiviral immunity where no natural resistance sources are known, relatively few genetically engineered virus-resistant crop plants have gained the necessary regulatory approval for use in commercial agriculture [12,23]. This is not because of lack of need, or lack of likely efficacy; it is more likely due to other factors, including the costs associated with intellectual property used for plant genetic engineering, governmental deregulation/approval costs and activities, and public perception of genetically engineered plants in general [24].

But where transgenic RNAi-based antivirus resistance has been used in agricultural systems so far, the benefits have been dramatic. One of the greatest success stories is the use of genetically engineered papaya ringspot virus (PRSV)-resistant papaya (Carica papaya), which was developed and used in Hawaii when no other options for controlling PRSV were available [25]. This essentially saved the Hawaiian papaya industry and today the transgenic virus-resistant papaya dominates the Hawaiian papaya market [26]. Another great benefit of RNAi-based transgenic resistance is that resistance can be simultaneously engineered toward more than one virus. This was first done and used successfully for the commercially grown Destiny III yellow crookneck squash (Cucurbita pepo) which was engineered to have robust, simultaneous RNAi resistance against two potyviruses and one cucumovirus (zucchini yellow mosaic virus, watermelon mosaic virus and cucumber mosaic virus, respectively) [27]. But a similar approach has now been used to confer resistance to cassava black streak disease (CBSD) in East and Central Africa, when essentially no other options were available. Cassava (Manihot esculenta) serves as a staple food crop for around $10 \%$ of the world's human population, many of whom are small subsistence farmers in tropical regions. Cassava is easy to grow and high in calories, but can be affected by various plant diseases, of which CBSD is currently the most 
threatening $[28,29]$. CBSD can be caused by either of two different but related viruses: cassava brown streak virus and Ugandan cassava brown streak virus [28]. Both viruses are widespread in cassava-growing regions, thus simultaneous resistance is needed to both. CBSD is so important that it has been listed as one of the seven plant disease threats to world food security [30]. Due to the importance of cassava as a local food source and CBSD as a disease, a multinational public-sector research effort (Virus Resistant Cassava for Africa) was established to use RNAi to develop CBSD-resistant cassava for use in Africa [31]. Beyene and colleagues engineered the locally desirable cassava cultivar, TME 204, with transgenes to generate hairpin RNAs corresponding to the coat protein coding regions of both viruses [32]. Subsequent greenhouse and field studies identified cassava lines that showed robust RNAi-based resistance to both viruses, and which was horticulturally desirable [32,33]. In May 2020, the Kenya Agricultural Research and Livestock Organization applied to the National Biosafety Authority for permission for open field cultivation and marketing of this transgenic CBSD-resistant cassava (https://cassavaplus.org/news/kalro-seeks-approval-to-introduce-disease-resistant-gm-cassava-2/). Thus RNAi offers great potential for controlling plant virus diseases and for helping to provide security for staple food crops.

\section{RNAi for plant disease control: more than just for viruses}

RNAi targets RNA. Most plant-infecting viruses have RNA as their genetic material and they replicate within the host cell, using its machinery to translate their mRNAs. If the plant cells are expressing interfering RNAs, the viral RNAs can be accessible for RNAi targeting. But all organisms use RNA to express their genetic information, so it seems that RNAi approaches might be used to target other types of plant pathogens and help to control the diseases they cause. However, unlike viruses, eukaryotic plant pathogens - including oomycetes, fungi, nematodes and parasitic plants - are in intimate contact with host cells, but the pathogen cell cytoplasm and that of the host plant remain physically separated. Still, RNAi activity is found in all eukaryotes, including eukaryotic plant pathogens. Therefore instead of targeting pathogen RNAs directly within the plant cell, as is the case for viruses, if RNAi inducers can be delivered from the plant into the cells of eukaryotic plant pathogens, there is an opportunity to induce RNAi effects within the pathogen as a strategy for plant disease control. In support of this conceptual approach, several recent studies have shown that small RNAs (including siRNAs and miRNAs) are mobile within plants [34]; they can have important roles in signaling and transfer from plants to cellular organisms and vice versa [35-37]; and, like dsRNAs, siRNAs and miRNAs can be inducers of RNAi. Finally, several studies have suggested that pathogen mRNAs for genes encoding necessary pathogenicity factors could be good RNAi targets.

Plant-to-pathogen transfer of RNAs that affect disease outcome has been shown to occur naturally in some plant-pathogen interactions. Verticillium dahliae is a soilborne fungus, it invades growing root tissues and causes Verticillium wilt of cotton (Gossypium hirsutum) [2]. Zhang and colleagues used small RNA sequencing to examine the small RNA populations in V. dahliae collected from infected cotton plants [38]. When they bioinformatically mapped these small RNAs back to the $V$. dahliae genome, they found that most mapped to $V$. dahliae but several mapped instead to the cotton genome; they concluded that these were produced in cotton but transferred into $V$. dahliae. Two of the small RNAs, miR159 and miR166, showed upregulated expression in cotton plants upon infection by $V$. dahliae and they were shown to target two V. dahliae mRNAs, for HiC-15 (a hydroxylase) and Clp-1 (a cysteine protease). They showed that both of these encoded proteins were important for $V$. dahliae virulence on cotton, and that virulence was reduced when the miRNAs targeted the respective mRNAs [38]. In another example, Cai et al. showed that $A$. thaliana cells secrete vesicle-encapsulated small RNAs which, when taken up by the fungal pathogen Botrytis cinerea, are able to induce RNAi effects in $B$. cinerea, targeting mRNAs critical for expression of pathogenicity genes [35]. Because in these examples the plant host is transferring the RNAi inducer molecules to the pathogen, this is commonly referred to as host-induced gene silencing (HIGS) [39]. HIGS is a natural means used by some plants to defend themselves against pathogens. It is also interesting to note that small RNA transfer goes both ways: some eukaryotic plant pathogens have been found to secrete small RNAs and/or proteins into host plants in order to interfere with plant RNAi defenses [36,37,40,41].

Transgenic HIGS approaches also have proven to be effective against several types of cellular plant pathogens, including fungi, oomycetes and nematodes (Figure 2) [42-45]. One of the first examples targeted the fungus Blumeria graminis, the causal agent of powdery mildew disease of many cereal crops. Powdery mildews are widespread diseases of many plants and are caused by various biotrophic fungi that can grow along and cover leaf surfaces of susceptible plant hosts [2]. The fungi produce specialized hyphae called haustoria that are intimately appressed to the host plant leaf epidermal cell plasma membranes, from which they absorb nutrients. Nowara and colleagues engineered barley (Hordeum vulgare) plants to produce hairpin RNAs homologous to the mRNAs for genes encoding pathogenicity factors (effectors Avra10 and Avrk1) in B. graminis [43]. When they inoculated the engineered plants with $B$. graminis, the plants showed reduced haustorium and fungal development; their interpretation was that the transgenic plant generated interfering RNAs (most likely siRNAs) which were available for uptake via $B$. graminis haustoria and were able to induce RNAi effects on the mRNA targets. Several other examples of using HIGS to target plant pathogenic fungi have also been reported [46].

Nematodes are round worms; many can be pathogens of plants. Some, such as the root knot nematode, Meloidogyne incognita, cause important economic crop losses in various plants worldwide [2]. Meloidogyne incognita feeds on plant roots, inducing gall formation and even plant death. Huang and colleagues engineered $A$. thaliana plants with hairpin constructs to induce RNAi effects against the $M$. incognita mRNA for the 16D10 peptide [44]. This peptide is a pathogenicity factor secreted into plant cells by root-feeding M. incognita. When $M$. incognita nematodes were allowed to feed on the engineered and non engineered $A$. thaliana plants, the engineered plants showed decreased gall development and fewer $M$. incognita eggs were produced [44]. Thus, the HIGS was effective against $M$. incognita. 
In contrast to eukaryotes, prokaryotes lack RNAi machinery; thus conventional RNAi approaches cannot be used to target prokaryotic plant pathogens. However, by understanding plant-pathogen interactions and the mechanisms associated with disease development, in some cases novel RNAi-based approaches are possible. As noted above, A. tumefaciens causes the disease crown gall, which can be a very important disease in many plants [2]. To induce crown gall development, $A$. tumefaciens transforms affected plant cells; it integrates its T-DNA into the plant cell chromosomal DNA, and the genes within the T-DNA - including those encoding for pathogenicity factors - are then transcribed by the plant cell to give mRNAs which will be translated by the plant cell translational machinery [14]. Dandekar and colleagues took a transgenic RNAi approach to target two of the mRNAs transcribed from the T-DNA as an approach to prevent crown gall development [47]. They engineered plants with hairpin constructs against the iaaM and ipt mRNAs that are derived from oncogenes found within the inserted T-DNA in infected plant cells. When plants were challenged with virulent $A$. tumefaciens, they saw $0-24 \%$ gall development in lines of the transgenic plants, and $100 \%$ gall development in non- engineered controls. Thus, when these plants were challenged by $A$. tumefaciens, transcription of the T-DNA-encoded oncogenes gave mRNAs which were degraded by RNAi activity already present in the plant cells.

\section{Non transgenic plant-based approaches for RNAi-mediated plant disease control}

The above examples and many more have shown that using transgenic plants for inducing RNAi activity against specific pathogens can give very effective control of plant diseases. However, recently other less labor-intensive, more flexible approaches have been demonstrated that do not require transgenic plants for RNAi-based protection against plant diseases. As noted above, viruses are powerful inducers of RNAi activity in plants. Fortunately, many viruses - even those with RNA genomes - have proved to be amenable for genetic engineering and it is possible to develop recombinant plant-infecting viruses for expressing desirable RNAs/proteins in plants. When the recombinant virus engineered to contain the RNAi inducer is inoculated to plants, RNAi activity will result, thereby generating an RNAi response (mostly siRNAs) against the infecting virus including the recombinant sequence (Figure 2).

Virus-mediated transient HIGS has been used to target various eukaryotic plant pathogens and even insect vectors of plant pathogens [12]. Barley stripe mosaic virus (BSMV) and tobacco rattle virus (TRV) are two plant viruses that can be engineered to express desired sequences in plants. BSMV was first used for HIGS in 2011 to target the wheat stripe rust fungus Puccinia striifomis $f$. sp. tritici. In this report, the BSMV-induced HIGS was found to slow the extension and development of fungal hyphae in infected plants [48]. BSMV has also been used to target Puccinia triticina and was found to reduce the fungal virulence in wheat by targeting mRNAs for fungal pathogenicity genes: MAPK, CYC1 and CNB [49]. BSMV containing antisense sequences against the mRNA targets of the fungal plant pathogen Fusarium culmorum, the causal agent of head blight in wheat, was shown to give a reduction of corresponding mRNA levels in the pathogen and reduced disease symptoms on wheat plants [50]. In the studies using TRV for HIGS induction, the authors first used recombinant TRV to target pathogenicity gene transcripts of $V$. dahliae and were able to suppress Verticillium wilt in tomato (Solanum lycopersicum) plants [51]. Based on their success, they then developed transgenic $A$. thaliana plants, also engineered to give stable HIGS against $V$. dahliae, and showed success [51].

Approximately $1 \%$ of the known flowering plants are parasites of other plants, and some are very important pathogens of their plant hosts [2]. For example, witchweed (Striga spp.) is widespread throughout sub-Saharan Africa and affects almost all cereal crops, causing losses ranging from 20 to 100\% [52]. These plant pathogens connect via haustoria to vascular tissues of their plant hosts, from which they obtain nutrients and/or water. Wuriyanghan and colleagues used TRV-based HIGS to identify pathogenicity genes in the holoparasitic plant Orobanche cumana [53]. They showed that HIGS targeting of pathogenicity gene transcripts reduced 0 . cumana growth and symptoms on the sunflower (Helianthus annus) host plants. Not only did they identify pathogenicity factors, but their results offer new potential applications for RNAi-based control of this important plant pathogen [53].

In addition to directly targeting plant pathogens, RNAi approaches have also shown good potential for targeting insects, including some that are vectors of plant pathogens (Figure 2) [12]. Wuriyanghan and Falk used recombinant tobacco mosaic virus to induce RNAi effects against mRNAs of the potato psyllid, Bactericera cockerelli, which is the vector for the bacterium Candidatus Liberibacter solanacearum, causal agent of zebra chip disease of tomatoes [54]. When $B$. cockerelli psyllids fed on plants infected with recombinant tobacco mosaic virus containing inserts homologous to $B$. cockerelli actin and V-ATPase mRNAs, RNAi effects were observed. They showed reductions in the corresponding mRNA targets, but also an important phenotypic effect: reduced fecundity when $B$. cockerelli reproduced on these plants. Similar efforts by others suggest that RNAi targeting of insect vectors of plant pathogens can give desired effects and can complement other approaches for plant disease control [55].

\section{Bacterium-mediated RNAi}

Bacteria have also been engineered to induce RNAi effects in their hosts. In 2001, the bacterium Escherichia coli was engineered and used to express and deliver dsRNAs to induce RNAi effects in Caenorhabditis elegans, the model experimental nematode [56]. This, of course, was one of the seminal studies and also led to a Nobel Prize, for discovery of the mechanism of RNAi. Similar bacterium-based efforts have subsequently been used for large-scale RNAi screens in various organisms [57]. Although it is still unclear how bacteria deliver and induce RNAi effects in their host organisms, it is known that bacteria can secrete various types of noncoding RNAs - also referred to as extracellular RNAs - by producing outer membrane vesicles. These vesicles may be relevant to bacterium-mediated RNAi because they have been known to contain small RNAs [58]. Because many bacteria are endosymbionts in plants and in insect vectors 
of plant pathogens, perhaps one approach could be to engineer the endosymbionts to express RNAi inducers in their hosts as a way to help control some plant diseases. One recent example supporting this concept is that of using bacterial endosymbiont-mediated RNAi targeting the tubulin gene transcripts of the western flower thrips (Frankliniella occidentalis), the insect vector of the widespread tomato spotted wilt virus [59]. Whitten and colleagues engineered the bacterial endosymbiont BFo2, eliminating its ability to produce RNase III, an enzyme that can degrade dsRNA [59]. Then they transformed BFo2 cells with a dsRNA expression cassette that targeted the $F$. occidentalis alpha-tubulin gene mRNAs, and introduced these bacteria back into $F$. occidentalis. This resulted in high mortality rates of F. occidentalis first-instar larvae within 4 days of infection and offered some protection to the plants used in the study. The conspecific spread of such dsRNA-producing endosymbiotic bacteria through an insect population represents an opportunity to produce sustained RNAi effects in target insects and novel opportunities for using RNAi in some plant diseases.

\section{Exogenous application of RNAi inducers to plants}

Research has shown that in some cases RNAi inducers (dsRNAs or even siRNAs) can be topically applied to plants, and either induce specific RNAi responses in the plant or are taken up by the plant and become available for delivery against target pathogens or their insect vectors $[13,60]$. This effect was first demonstrated against plant viruses [61] and since then has been shown to be effective in targeting a variety of plant pathogens, including various plant viruses and viroids, fungal pathogens and even insect vectors of plant pathogens [13]. Topical applications of RNAi inducers have shown success against two unrelated plant pathogenic fungi, $B$. cinerea and Fusarium graminearum [62,63]. Botrytis cinerea mycelia could take up both externally applied siRNAs and dsRNAs, resulting in transcript reductions for the $B$. cinerea Dicer-like 1 (Bc-DCL1) and Bc-DCL2 genes. The reduced accumulation of $B c-D C L 1$ and $B c-D C L 2$ transcripts resulted in attenuated $B$. cinerea pathogenicity in $A$. thaliana and tomato plants. Topical applications of RNAi inducers also proved to be effective for impacting $F$. graminearum infections on barley ( $H$. vulgare) plants [64]. Koch and colleagues have previously shown that a spray application of long noncoding dsRNAs (targeting transcripts for the three fungal genes required for biosynthesis of fungal ergosterol) inhibited F. graminearum growth not only in the directly sprayed leaf tissues, but also in the non sprayed parts of detached leaves. It was shown that the effects in the non sprayed distal tissues required the transport of the dsRNAs and/or siRNAs through the plant vasculature, followed by uptake by F. graminearum from the plant [64].

Topical applications of RNAi inducers (mostly dsRNAs) show interesting potential for plant disease control, but several factors must be overcome for translational application. One is that plant cells are covered by cellulosic cell walls, and epidermal tissues often have waxy coverings, making direct application and uptake of RNAs somewhat problematic $[65,66]$. It has been shown experimentally that high-pressure sprays increased the efficacy of delivering interfering RNAs to plants [66], and efforts are ongoing to develop compounds that will aid in uptake and transport and increase efficacy of topical applications of interfering RNAs [65]. A second problematic factor is that RNAs are not stable molecules when exposed in the environment, such as on the surfaces of plant leaves. Although long dsRNA is relatively more stable than other forms of RNA, dsRNAs are still susceptible to degradation. Strategies to increase the stability and longevity of dsRNAs for topical applications to plants can be improved by approaches such as complexing dsRNAs with bioclays (doublelayered hydroxide nanosheets) [60,67]. In one study, dsRNA bioclays were shown to give up to 20 days' protection from viral infection to plants subjected to mechanical virus inoculation, compared with only 5 days' protection for plants treated with naked dsRNAs. This technology provided some protection to the dsRNAs, but also provided a slower release of the dsRNAs into the plant, thereby inducing RNAi effects over a longer time [67]. These studies showed that the topical application of dsRNAs could provide some level of protection for plants from various pathogens; however, the mechanisms for uptake of dsRNAs, and whether dsRNAs or siRNAs are transported in plants, are still unclear.

\section{Conclusion \& future perspective}

In plants, naturally occurring RNAi pathways affect gene regulation, cross-kingdom communication between organisms and antiviral defense. However, through understanding RNAi pathways and the factors involved, many labs have been able to exploit some aspects of RNAi interactions in plants to gain novel opportunities for plant disease control. Transgenic and non-transgenic plant-based RNAi approaches for different pathogens in different plants have shown great promise, and new opportunities are undoubtedly coming. Several recent publications discuss the potential additional applications, marketability and environmental risk considerations relative to practical use of RNAi strategies in agriculture [68-70].

Where transgenic plant RNAi-based virus resistance has so far been used in commercial agriculture, the economic and environmental benefits are clear. In Hawaii, transgenic, disease-resistant papayas saved the industry [26]; they now make up the majority of the market, they are exported to other countries for consumption and, interestingly, they are even grown to provide a protective PRSV-free buffer around non transgenic, PRSV-susceptible papayas, thereby allowing commercial production of the PRSV-susceptible varieties [71]. When potatoes with transgenic resistance to potato leafroll virus (PLRV) were grown in the US Pacific Northwest in 2004, estimates suggested that farmers saved \$141-164 per acre versus the costs associated with attempting to protect susceptible potatoes [72]. The authors estimated that if $80 \%$ of the potatoes grown in the region had RNAi-based resistance to PLRV, this could result in a reduction of approximately 1.8 million pounds of insecticides used on potatoes per year, because farmers would no longer need to spray to control aphid vectors of PLRV. We now know also that in addition to targeting viruses, RNAi-based resistance can be effective against cellular plant pathogens and even insect pests. RNAi approaches against viruses directly target the virus RNAs within the host plant cell, while RNAi approaches 
against cellular pathogens mostly target mRNAs for pathogen-encoded pathogenicity factors in the pathogen, and the RNAi inducers are most likely delivered into the pathogen via plant-to-pathogen small RNA (mostly siRNA) exchange events.

With rapidly improving sequencing technologies, RNAi effects are being discovered and studied more comprehensively in many different organisms. The discovery and new knowledge gained will open up new opportunities for developing novel and more extensive approaches that can contribute to plant disease control, improve agriculture in general and help feed the planet's increasing human population.

\section{Acknowledgments}

The authors thank the anonymous reviewers for their constructive suggestions to this work.

\section{Financial \& competing interests disclosure}

The authors have no relevant affiliations or financial involvement with any organization or entity with a financial interest in or financial conflict with the subject matter or materials discussed in the manuscript. This includes employment, consultancies, honoraria, stock ownership or options, expert testimony, grants or patents received or pending, or royalties.

No writing assistance was utilized in the production of this manuscript.

\section{Open access}

This work is licensed under the Attribution-NonCommercial-NoDerivatives 4.0 Unported License. To view a copy of this license, visit http://creativecommons.org/licenses/by-nc-nd/4.0/

\section{References}

1. Velasquez AC, Castroverde CDM, He SY. Plant-pathogen warfare under changing climate conditions. Curr. Biol. 28(10), R619-R634 (2018).

2. Schumann GL, D'arcy CJ. Essential Plant Pathology. The American Phytopathological Society, MN, USA (2010).

3. Willocquet L, Savary S, Yuen J. Multiscale phenotyping and decision strategies in breeding for resistance. Trends Plant Sci. 22(5), 420-432 (2017).

4. Mcdonald BA. Using dynamic diversity to achieve durable disease resistance in agricultural ecosystems. Tropical Plant Pathol. 39, 191-196 (2014).

5. Brown JK. Durable resistance of crops to disease: a Darwinian perspective. Annu. Rev. Phytopathol. 53, 513-539 (2015).

6. Muhammad T, Zhang F, Zhang Y, Liang Y. RNA interference: a natural immune system of plants to counteract biotic stressors. Cells 8(1), 38 (2019).

7. Gasciolli V, Mallory AC, Bartel DP, Vaucheret H. Partially redundant functions of Arabidopsis DICER-like enzymes and a role for DCL4 in producing trans-acting siRNAs. Curr. Biol. 15(16), 1494-1500 (2005)

8. Baulcombe DC. How virus resistance provided a mechanistic foundation for RNA silencing. Plant Cell 31(7), 1395-1396 (2019).

9. Dalmay T, Hamilton A, Rudd S, Angell S, Baulcombe DC. An RNA-dependent RNA polymerase gene in Arabidopsis is required for posttranscriptional gene silencing mediated by a transgene but not by a virus. Cell 101(5), 543-553 (2000).

10. Budak H, Akpinar BA. Plant miRNAs: biogenesis, organization and origins. Funct. Integr. Genomics 15(5), 523-531 (2015).

11. Guo Z, Li Y, Ding SW. Small RNA-based antimicrobial immunity. Nat. Rev. Immunol. 19(1), 31-44 (2019).

12. Rosa C, Kuo YW, Wuriyanghan H, Falk BW. RNA interference mechanisms and applications in plant pathology. Annu. Rev. Phytopathol. 56, 581-610 (2018).

13. Dalakouras A, Wassenegger M, Dadami E, Ganopoulos I, Pappas ML, Papadopoulou K. Genetically modified organism-free RNA interference: exogenous application of RNA molecules in plants. Plant Physiol. 182(1), 38-50 (2020).

14. Lacroix B, Citovsky V. Pathways of DNA transfer to plants from Agrobacterium tumefaciens and related bacterial species. Annu. Rev. Phytopathol. 57, 231-251 (2019).

15. Horsch RB, Fry JE, Hoffmann NL, Eichholtz D, Rogers SG, Fraley RT. A simple and general method for transferring genes into plants. Science 227, 1229-1231 (1985).

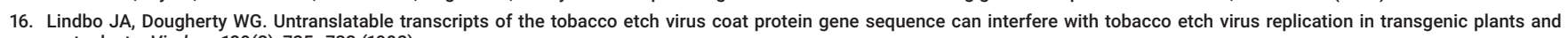
protoplasts. Virology 189(2), 725-733 (1992)

17. Lindbo JA, Dougherty WG. Pathogen-derived resistance to a potyvirus: immune and resistant phenotypes in transgenic tobacco expressing altered forms of a potyvirus coat protein nucleotide sequence. Mol. Plant Microbe Interact. 5(2), 144-153 (1992).

18. Lindbo JA, Silva-Rosales L, Proebsting WM, Dougherty WG. Induction of a highly specific antiviral state in transgenic plants: implications for regulation of gene expression and virus resistance. Plant Cell 5(12), 1749-1759 (1993).

19. Lindbo JA, Falk BW. The impact of 'coat protein-mediated virus resistance' in applied plant pathology and basic research. Phytopathology 107(6), 624-634 (2017).

20. Fire A, Xu S, Montgomery MK, Kostas SA, Driver SE, Mello CC. Potent and specific genetic interference by double-stranded RNA in Caenorhabditis elegans. Nature 391(6669), 806-811 (1998).

21. Wang MB, Abbott DC, Waterhouse PM. A single copy of a virus-derived transgene encoding hairpin RNA gives immunity to barley yellow dwarf virus. Mol. Plant Pathol. 1(6), 347-356 (2000).

22. Waterhouse PM, Graham MW, Wang MB. Virus resistance and gene silencing in plants can be induced by simultaneous expression of sense and antisense RNA. Proc. Natl Acad. Sci. USA 95(23), 13959-13964 (1998)

23. Dong OX, Ronald PC. Genetic engineering for disease resistance in plants: recent progress and future perspectives. Plant Physiol. 180(1), 26-38 (2019).

24. Pixley KV, Falck-Zepeda JB, Giller KE et al. Genome editing, gene drives, and synthetic biology: will they contribute to disease-resistant crops, and who will benefit? Annu. Rev. Phytopathol. 57, 165-188 (2019)

25. Gonsalves D. Transgenic papaya: development, release, impact and challenges. Adv. Virus Res. 67, 317-354 (2006).

26. Business Insider. GMO papaya saved an $\$ 11$ million industry in Hawaii - and set off a political storm. https://geneticliteracyproject.org/2017/06/26/gmo-papayas-saved-11-million-indus try-set-off-political-storm-hawaii/

27. Fuchs M, Tricoli DM, Carney KJ, Schesser M, Mcferson JR, Gonsalves D. Comparative virus resistance and fruit yield of transgenic squash with single and multiple coat protein genes. Plant Dis. 82(12), 1350-1356 (1998).

28. Patil BL, Legg JP, Kanju E, Fauquet CM. Cassava brown streak disease: a threat to food security in Africa. J. Gen. Virol. 96(5), 956-968 (2015).

29. Gomez MA, Lin ZD, Moll T et al. Simultaneous CRISPR/Cas9-mediated editing of cassava elF4E isoforms nCBP-1 and nCBP-2 reduces cassava brown streak disease symptom severity and incidence. Plant Biotechnol. J. 17(2), 421-434 (2019).

30. Pennisi E. Armed and dangerous. Science 327(5967), 804-805 (2010).

31. Taylor NJ, Halsey M, Gaitan-Solis E et al. The VIRCA Project: virus resistant cassava for Africa. GM Crops Food 3(2), 93-103 (2012).

32. Beyene G, Chauhan RD, llyas M et al. A virus-derived stacked RNAi construct confers robust resistance to cassava brown streak disease. Front. Plant Sci. 7, 2052 (2016).

33. Wagaba H, Beyene G, Aleu J et al. Field level RNAi-mediated resistance to cassava brown streak disease across multiple cropping cycles and diverse East African agro-ecological locations. Front. Plant Sci. 7, 2060 (2016) 
34. Ham BK, Lucas WJ. Phloem-mobile RNAs as systemic signaling agents. Annu. Rev. Plant Biol. 68, 173-195 (2017).

35. Cai Q, Qiao L, Wang M et al. Plants send small RNAs in extracellular vesicles to fungal pathogen to silence virulence genes. Science 360(6393), 1126-1129 (2018).

36. Hudzik C, Hou Y, Ma W, Axtell MJ. Exchange of small regulatory RNAs between plants and their pests. Plant Physiol. 182(1), 51-62 (2020).

37. Knip M, Constantin ME, Thordal-Christensen H. Trans-kingdom cross-talk: small RNAs on the move. PLoS Genet. 10(9), e1004602 (2014).

38. Zhang T, Zhao YL, Zhao JH et al. Cotton plants export microRNAs to inhibit virulence gene expression in a fungal pathogen. Nat. Plants 2(10), 16153 (2016).

39. Baulcombe DC. VIGS, HIGS and FIGS: small RNA silencing in the interactions of viruses or filamentous organisms with their plant hosts. Curr. Opin. Plant Biol. 26, 141-146 (2015).

40. Qiao Y, Liu L, Xiong Q et al. Oomycete pathogens encode RNA silencing suppressors. Nat. Genet. 45(3), 330-333 (2013).

41. Weiberg A, Wang M, Bellinger M, Jin H. Small RNAs: a new paradigm in plant-microbe interactions. Annu. Rev. Phytopathol. 52, 495-516 (2014).

42. Tinoco ML, Dias BB, Dall'astta RC, Pamphile JA, Aragao FJ. In vivo trans-specific gene silencing in fungal cells by in planta expression of a double-stranded RNA. BMC Biol. 8, 27 (2010).

43. Nowara D, Gay A, Lacomme C et al. HIGS: host-induced gene silencing in the obligate biotrophic fungal pathogen Blumeria graminis. Plant Cell 22(9), 3130-3141 (2010).

44. Huang G, Allen R, Davis EL, Baum TJ, Hussey RS. Engineering broad root-knot resistance in transgenic plants by RNAi silencing of a conserved and essential root-knot nematode parasitism gene. Proc. Natl Acad. Sci. USA 103(39), 14302-14306 (2006).

45. Jahan SN, Asman AK, Corcoran P, Fogelqvist J, Vetukuri RR, Dixelius C. Plant-mediated gene silencing restricts growth of the potato late blight pathogen Phytophthora infestans. J. Exp. Bot. 66(9), 2785-2794 (2015).

46. Sang H, Kim Jl. Advanced strategies to control plant pathogenic fungi by host-induced gene silencing (HIGS) and spray-induced gene silencing (SIGS). Plant Biotechnol. Rep. 14, 1-8 (2020).

47. Escobar MA, Civerolo EL, Summerfelt KR, Dandekar AM. RNAi-mediated oncogene silencing confers resistance to crown gall tumorigenesis. Proc. Nat/ Acad. Sci. USA 98(23), 13437-13442 (2001).

48. Yin C, Jurgenson JE, Hulbert SH. Development of a host-induced RNAi system in the wheat stripe rust fungus Puccinia striiformis $f$. sp. tritici. Mol. Plant Microbe Interact. 24(5), 554-561 (2011).

49. Panwar V, Mccallum B, Bakkeren G. Host-induced gene silencing of wheat leaf rust fungus Puccinia triticina pathogenicity genes mediated by the barley stripe mosaic virus. Plant Mol. Biol. 81(6), 595-608 (2013).

50. Chen W, Kastner C, Nowara D et al. Host-induced silencing of Fusarium culmorum genes protects wheat from infection. J. Exp. Bot. 67(17), 4979-4991 (2016).

51. Song Y, Thomma B. Host-induced gene silencing compromises Verticillium wilt in tomato and Arabidopsis. Mol. Plant Pathol. 19(1), 77-89 (2018).

52. Runo S, Kuria EK. Habits of a highly successful cereal killer, Striga. PLoS Pathog. 14(1), e1006731 (2018).

53. Jiang J, Zhao Q, Bai R et al. Host sunflower induced silencing of parasitism-related genes confers resistance to invading Orobanche cumana. Plant Physiol. (In Press).

54. Wuriyanghan H, Falk BW. RNA Interference towards the potato psyllid, Bactericera cockerelli, is induced in plants infected with recombinant tobacco mosaic virus (TMV). PLoS ONE 8(6), e66050 (2013).

55. Hajeri S, Killiny N, El-Mohtar C, Dawson WO, Gowda S. Citrus tristeza virus-based RNAi in citrus plants induces gene silencing in Diaphorina citri, a phloem-sap sucking insect vector of citrus greening disease (Huanglongbing). J. Biotechnol. 176, 42-49 (2014).

56. Timmons L, Court DL, Fire A. Ingestion of bacterially expressed dsRNAs can produce specific and potent genetic interference in Caenorhabditis elegans. Gene 263(1-2), 103-112 (2001).

57. Newmark PA, Reddien PW, Cebria F, Sanchez Alvarado A. Ingestion of bacterially expressed double-stranded RNA inhibits gene expression in planarians. Proc. Natl Acad. Sci. USA 100(Suppl. 1), 11861-11865 (2003).

58. Baldrich P, Rutter BD, Karimi HZ, Podicheti R, Meyers BC, Innes RW. Plant extracellular vesicles contain diverse small RNA species and are enriched in 10- to 17-nucleotide 'tiny' RNAs. Plant Cell 31(2), 315-324 (2019).

59. Whitten MM, Facey PD, Del Sol R et al. Symbiont-mediated RNA interference in insects. Proc. Biol. Sci. 283(1825), 20160042 (2016).

60. Fletcher SJ, Reeves PT, Hoang BT, Mitter N. A perspective on RNAi-based biopesticides. Front. Plant Sci. 11, 51 (2020).

61. Tenllado F, Diaz-Ruiz JR. Double-stranded RNA-mediated interference with plant virus infection. J. Virol. 75(24), 12288-12297 (2001).

62. Wang M, Weiberg A, Lin FM, Thomma BP, Huang HD, Jin H. Bidirectional cross-kingdom RNAi and fungal uptake of external RNAs confer plant protection. Nat. Plants 2, 16151 (2016).

63. Ostry V, Ovesna J, Skarkova J, Pouchova V, Ruprich J. A review on comparative data concerning Fusarium mycotoxins in Bt maize and non-Bt isogenic maize. Mycotoxin Res. 26(3), $141-145$ (2010).

64. Koch A, Biedenkopf D, Furch A et al. An RNAi-based control of Fusarium graminearum infections through spraying of long dsRNAs involves a plant passage and is controlled by the fungal silencing machinery. PLoS Pathog. 12(10), e1005901 (2016).

65. Bennett M, Deikman J, Hendrix B, landolino A. Barriers to efficient foliar uptake of dsRNA and molecular barriers to dsRNA activity in plant cells. Front. Plant Sci. 11, 816 (2020).

66. Dalakouras A, Wassenegger M, Mcmillan JN et al. Induction of silencing in plants by high-pressure spraying of in vitro-synthesized small RNAs. Front. Plant Sci. 7, 1327 (2016).

67. Mitter N, Worrall EA, Robinson KE et al. Clay nanosheets for topical delivery of RNAi for sustained protection against plant viruses. Nat. Plants 3 , 16207 (2017).

68. Christiaens O, Dzhambazova T, Kostov K et al. Literature review of baseline information on RNAi to support the environmental risk assessment of RNAi-based GM plants. European Food Safety Agency doi:10.2903/sp.efsa.2018.EN-1424 2018) (Epub ahead of print).

69. Mezzetti B, Smagghe G, Arpaia S et al. RNAi: what is its position in agriculture? J. Pest Sci. 93, 1125-1130 (2020).

70. Taning CN, Arpaia S, Christiaens 0 et al. RNA-based biocontrol compounds: current status and perspectives to reach the market. Pest Manag. Sci. 76(3), 841-845 (2020).

71. Fitch MMM. Update on gene transfer biotechnology of papaya. Acta Hort. 1111, 7-18 (2016).

72. Kaniewski WK, Thomas PE. The potato story. AgBioForum 7, 41-46 (2004). 
